\title{
Strategyproof Mechanism for Two Heterogeneous Facilities with Constant Approximation Ratio
}

\author{
Minming $\mathbf{L i}^{1}$, Pinyan $\mathbf{L u}^{2}$, Yuhao Yao ${ }^{3}$ and Jialin Zhang ${ }^{3,4}$ \\ ${ }^{1}$ City University of Hong Kong, Hong Kong, China \\ ${ }^{2}$ ITCS, Shanghai University of Finance and Economics, Shanghai, China \\ ${ }^{3}$ University of Chinese Academy of Sciences, Beijing, China \\ ${ }^{4}$ Institute of Computing Technology, Chinese Academy of Sciences, Beijing, China \\ minming.li@cityu.edu.hk, lu.pinyan@mail.shufe.edu.cn, yaoyuhao15@mails.ucas.ac.cn, \\ zhangjialin@ict.ac.cn
}

\begin{abstract}
In this paper, we study the two-facility location game with optional preference where the acceptable set of facilities for each agent could be different and an agent's cost is his distance to the closest facility within his acceptable set. The objective is to minimize the total cost of all agents while achieving strategyproofness. For general metrics, we design a deterministic strategyproof mechanism for the problem with approximation ratio of $1+2 \alpha$, where $\alpha$ is the approximation ratio of the offline optimization version. In particular, for the setting on a line, we improve the earlier best ratio of $n / 2+1$ [Yuan et al., 2016] to a ratio of 2.75 .
\end{abstract}

\section{Introduction}

Facility location games have been studied extensively in recent years. In the general setting, an authority adopts a mechanism to decide where to place the facilities in the existence of participating agents with their reported locations or preferences. The authority aims to minimize the social cost on the condition that agents will not gain by misreporting their information. This property is called strategyproofness. The cost of an agent is defined as his distance to the facility. In the most classic setting for single facility on a line and private location information of agents, the median mechanism is strategyproof and minimizes the social cost [Moulin, 1980]. Procaccia and Tennenholtz introduced the setting for twofacility location games and initiated the approximate mechanism design for facility location games [Procaccia and Tennenholtz, 2009]. In this two-facility model, an agent's cost is his distance to the closer facility. Unlike the single facility setting, the optimality no longer coincides with strategyproofness in this model. They proved constant lower bound for the approximation ratio and provide a strategyproof mechanism with linear $O(n)$ approximation ratio. This left a huge gap for the ratio. Lu et al. obtained a randomized mechanism which achieves a constant approximation ratio of 4 and proved a linear lower bound $\Omega(n)$ for all deterministic strategyproof mechanisms [Lu et al., 2010].

In their model, two facilities are identical and an agent is interested in the one closer to him. For two heterogeneous facilities, some agent may only accept one of them and the cost is his distance to that particular one. Of course, if every agent is only interested in one of them, it becomes two separate single facility games and the solutions become trivial. The interesting setting is that some agents are interested in both facilities while others are interested in only one of them, and these preferences are agents' private information. In general, an agent's cost is his distance to the closest facility in his acceptable set. This heterogeneous facilities model was proposed in [Yuan et al., 2016]. To study the effect of private preference, they assumed that the location information is public in this new model. This is also a more realistic model since in many real facility location applications, agents' locations can be publicly verified.

In their paper, they proved a lower bound of 2 and provided a strategyproof mechanism with linear $O(n)$ approximation ratio. This again left a huge gap for the ratio. It is also not clear whether there is a similar large gap between randomized and deterministic mechanisms as the homogeneous two-facility setting.

Besides its application relevance, the facility location game is also a very important playground for the theory of strategyproof mechanisms without money. Due to the remarkable Arrow's impossibility theorem [Arrow, 1950] and GibbardSatterthwaite's impossibility theorem [Gibbard, 1973; Satterthwaite, 1975], the space of strategyproof mechanisms is very limited. Gibbard-Satterthwaite's impossibility theorem states that any strategyproof social choice function onto a set of alternatives with at least three options is a dictatorship in the general preference domain. Basically, there are only two types of strategyproof mechanisms: dictatorship or majority voting on two options. The median mechanism is neither of them. The existence of such non trivial strategyproof mechanisms is due to single-peaked preference structure of the problem while the Gibbard-Satterthwaite's impossibility theorem is for general preferences. Due to this, single-peaked preference is an important direction in the study of strategyproof mechanisms without money. Studying other variants of facility location games may lead to the discovery of other interesting preference structures and non trivial strategyproof mechanisms. In most extended version of facility location games, the proposed strategyproof mechanisms are some ver- 
sions (maybe randomized) of dictatorship, a majority voting of two boundary options or things like that. The proportional mechanism from [Lu et al., 2010] is one notably nontrivial example. But this one only satisfies a weaker version of strategyproofness, namely strategyproofness in expectation. This might be the reason why there is a huge gap between randomized and deterministic mechanisms. It would be more interesting if there are other nontrivial deterministic mechanisms.

\subsection{Our Contribution}

In this paper, we design a novel strategyproof mechanism which achieves an approximation ratio of $1+2 \alpha$, where $\alpha$ is the approximation ratio of the offline optimization version. If we do not care about computational complexity or the offline optimization problem is polynomial time solvable such as the line metric case, this is a 3-approximation strategyproof mechanism. This asymptotically resolves the problem of the two heterogeneous facilities game. Our mechanism is very simple. We first ignore agents' preferences and assume that both facilities are acceptable for each agent. We then, purely based on the public location information of agents, compute the optimal locations of two facilities, which we call $s_{1}$ and $s_{2}$. On a line, the optimal location can be computed in polynomial time. Then among four possible locations of the two facilities $\left(s_{1}, s_{1}\right),\left(s_{1}, s_{2}\right),\left(s_{2}, s_{1}\right),\left(s_{2}, s_{2}\right)$, we choose the one with the minimum social cost based on agents' reported preferences.

For the special case of line metric, we further improve the analysis and show that the same mechanism is of 2.75approximation. On the other hand, we construct an example to show that the approximation ratio of our mechanism is at least $1+\sqrt{2}$ and conjecture that this lower bound is tight.

Our mechanism is deterministic and very simple but at the same time highly non-trivial in the sense that its strategyproofness requires an interesting argument. It does not belong to the two exception families of Gibbard-Satterthwaite's impossibility theorem. The reason for the median mechanism being strategyproof is the single-peaked preference. It is an interesting open direction to figure out what kind of preference structure makes our mechanism strategyproof. This may lead to a generalized version of our mechanism, which works for a family of problems.

It is natural to think about the extension of our mechanism for the facility location game with three or more facilities. However, there is an example to show that the mechanism is no longer strategyproof even for line metric. The situation is similar to what happens in the proportional mechanism [Lu et al., 2010], whose three-facility version is no longer strategyproof. It is an interesting open question to extend our result to the facility location game with three or more facilities. We note that the same problem for homogeneous facilities remains open after almost a decade.

\subsection{Related Works}

There are two major perspectives to approach the facility location game, namely, characterizing strategyproof mechanisms and designing strategyproof mechanisms. The classic agent preference for facility location games on a line is a special case of single-peaked preference for which [Moulin, 1980] characterized all the anonymous, strategyproof and efficient mechanisms and at the same time showed that median mechanism is strategyproof for minimizing social cost. This is the pioneering work in the field. Later on, more characterizations are studied on networks [Schummer and Vohra, 2002], for two facilities [Fotakis and Tzamos, 2014] and for double-peaked preference [Filos-Ratsikas et al., 2017]. There are also characterizations for other variants of facility location games.

When optimality no longer coincides with strategyproofness, Procaccia and Tennenholtz initiated the approximate mechanism design for facility location games [Procaccia and Tennenholtz, 2009]. Since then, facility location games are enriched from many different perspectives.

In terms of preference of agents for a single facility, [Cheng et al., 2011] initiated obnoxious facility location games where agents want to be far away from the facility, followed by [Ibara and Nagamochi, 2012; Oomine and Nagamochi, 2016; Mei et al., 2018b]. [Zou and Li, 2015] and [Feigenbaum and Sethuraman, 2015] studied dual preference where agents have different preferences towards the single facility. [FilosRatsikas et al., 2017] studied double-peaked preference.

In terms of the agents' cost function with respect to the distance to the facility, besides linear functions, there are also other functions studied. For example, threshold based linear function [Mei et al., 2018a], agent dependent linear function (happiness) [Mei et al., 2016] and concave function [Fotakis and Tzamos, 2016].

In terms of social objective, besides the mostly studied social cost/utility and maximum cost/utility, there are also other objectives studied like sum of square of distances[Feldman and Wilf, 2013; Mei et al., 2018b], difference of maximum distance and minimum distance (envy) [Cai et al., 2016].

The studies on heterogeneous facilities was initiated by [Serafino and Ventre, 2014; Serafino and Ventre, 2015], where the cost of an agent who likes both facilities is the sum of the distances to both facilities. When the cost is only affected by the closer facility or the farther facility, it is called optional preference and is studied by [Yuan et al., 2016]. Recently fractional preference is studied by [Fong et al., 2018], where the cost is a weighted sum of the two distances. There are also works setting a distance constraint between the two facilities, either maximum distance [Zou and Li, 2015; Chen et al., 2018] or minimum distance [Duan et al., 2019].

For other metric spaces, [Alon et al., 2010] and [Cheng et al., 2013] studied networks. [Lu et al., 2010] studied Euclidean space where a 4-approximated randomized strategyproof mechanism is proposed.

\section{Preliminaries}

There are $n$ agents in a metric space with a distance metric $d()$ and the government is to build two facilities (named as $F_{1}$ and $F_{2}$ ) for them. Agent $i$ is located at $x_{i}$ and has a preference $p_{i} \in\left\{\left\{F_{1}\right\},\left\{F_{2}\right\},\left\{F_{1}, F_{2}\right\}\right\}$. Here, we call $\boldsymbol{x}=$ $\left\{x_{1}, x_{2}, \ldots, x_{n}\right\}$ as location profile and $\boldsymbol{p}=\left\{p_{1}, p_{2}, \ldots, p_{n}\right\}$ as preference profile.

Given that $F_{1}$ is built at $y_{1}$ and $F_{2}$ is built at $y_{2}$, we define 
the cost of agent $i$ as $c_{\boldsymbol{x}, \boldsymbol{p}, i}\left(y_{1}, y_{2}\right)=\min _{k: F_{k} \in p_{i}} d\left(x_{i}, y_{k}\right)$. Here, we denote by $d(x, y)$ the distance between $x$ and $y$ in the metric space. The social cost is defined as $S_{\boldsymbol{x}, \boldsymbol{p}}\left(y_{1}, y_{2}\right)$ $=\sum_{i=1}^{n} c_{\boldsymbol{x}, \boldsymbol{p}, i}\left(y_{1}, y_{2}\right)$.

In this model, the government uses a mechanism to decide the places to build the facilities. More specifically, a mechanism takes a location profile $\boldsymbol{x}$ and a preference profile $\boldsymbol{p}$ as input and outputs facilities' locations.

Given that an agent might misreport his preference, which will lead to a different output by the mechanism and might lower his own cost, the government needs to design a strategyproof mechanism to avoid this situation.

Definition 1. (strategyproofness)

A mechanism $M$ is strategyproof if for any agent, cheating will not decrease her cost. Formally, for any location and preference profile $\boldsymbol{x}$ and $\boldsymbol{p}$ and any agent $i$ if he cheats by misreporting his preference as $p_{i}^{\prime}$ and the preference profile changes to $\boldsymbol{p}^{\prime}=\left\{p_{1}, \ldots, p_{i-1}, p_{i}^{\prime}, p_{i+1}, \ldots, p_{n}\right\}$, then there must be $c_{\boldsymbol{x}, \boldsymbol{p}, i}(M(\boldsymbol{x}, \boldsymbol{p})) \leq c_{\boldsymbol{x}, \boldsymbol{p}, i}\left(M\left(\boldsymbol{x}, \boldsymbol{p}^{\prime}\right)\right)$.

Given that no strategyproof mechanism achieves the minimum social cost [Yuan et al., 2016], we use approximation ratio to evaluate the performance of mechanisms.

Definition 2. (approximation ratio)

A mechanism $M$ has an approximation ratio of $\gamma$ if for any location and preference profile $\boldsymbol{x}$ and $\boldsymbol{p}, S C_{\boldsymbol{x}, \boldsymbol{p}}(M(\boldsymbol{x}, \boldsymbol{p})) \leq$ $\gamma \cdot \min _{y_{1}, y_{2}} S C_{\boldsymbol{x}, \boldsymbol{p}}\left(y_{1}, y_{2}\right)$.

\section{Our Strategyproof Mechanism}

In this section, we introduce our mechanism and prove its strategyproofness. In Section 4, we will analyze its approximation ratio.

\subsection{The Mechanism}

Mechanism 1. Denote by $\boldsymbol{q}$ the preference profile $\left\{\left\{F_{1}, F_{2}\right\},\left\{F_{1}, F_{2}\right\}, \ldots,\left\{F_{1}, F_{2}\right\}\right\}$, i.e. every agent prefers both facilities. Let $A$ be the set of all available facility locations and let location pair $\left(s_{1}, s_{2}\right)$ satisfy $\left(s_{1}, s_{2}\right)=$ $\arg \min _{y_{1}, y_{2} \in A} S C_{\boldsymbol{x}, \boldsymbol{q}}\left(y_{1}, y_{2}\right)$. Finally, we output the pair $\left(f_{1}, f_{2}\right)=\arg \min _{y_{1}, y_{2} \in\left\{s_{1}, s_{2}\right\}} S C_{\boldsymbol{x}, \boldsymbol{p}}\left(y_{1}, y_{2}\right)$. We break the tie in this order: $\left(s_{1}, s_{1}\right),\left(s_{1}, s_{2}\right),\left(s_{2}, s_{1}\right),\left(s_{2}, s_{2}\right)$.

Actually, it is a special case of the $k$-median problem to calculate $s_{1}$ and $s_{2}$, i.e. the case in which $k$ is equal to 2 . When $|A|$ is finite, we can enumerate the locations of $s_{1}$ and $s_{2}$ in $O\left(|A|^{2}\right)$ time. However, it might be hard when $|A|$ is not finite and we may only use some approximation algorithm to solve the problem. For instance, [Arora et al., 1998] provides a PTAS for $k$-median for Euclidean space $\mathbb{R}^{2}$.

In the rest of this paper, we denote by $s_{1}^{\prime}$ and $s_{2}^{\prime}$ the result calculated by the 2-median problem approximation oracle with approximation ratio $\alpha$.

\subsection{Strategyproofness}

\section{Theorem 1. Mechanism 1 is strategyproof.}

Proof. By the definition of Mechanism 1, we can see that $s_{1}^{\prime}$ and $s_{2}^{\prime}$ do not rely on agents' preferences and the two facilities' locations will always be one among $\left(s_{1}^{\prime}, s_{1}^{\prime}\right),\left(s_{1}^{\prime}, s_{2}^{\prime}\right)$, $\left(s_{2}^{\prime}, s_{1}^{\prime}\right)$ and $\left(s_{2}^{\prime}, s_{2}^{\prime}\right)$.

\begin{tabular}{|c|c|}
\hline$\left(y_{1}, y_{2}\right)$ & $S C_{\boldsymbol{x}, \boldsymbol{p}^{\prime}}\left(y_{1}, y_{2}\right)-S C_{\boldsymbol{x}, \boldsymbol{p}}\left(y_{1}, y_{2}\right)$ \\
\hline$\left(s_{1}^{\prime}, s_{1}^{\prime}\right)$ & 0 \\
\hline$\left(s_{1}^{\prime}, s_{2}^{\prime}\right)$ & $d\left(x_{i}, s_{1}^{\prime}\right)-d\left(x_{i}, s_{2}^{\prime}\right) \geq 0$ \\
\hline$\left(s_{2}^{\prime}, s_{1}^{\prime}\right)$ & 0 \\
\hline$\left(s_{2}^{\prime}, s_{2}^{\prime}\right)$ & 0 \\
\hline
\end{tabular}

Table 1: the difference in social cost for $\boldsymbol{p}^{\prime}$ and $\boldsymbol{p}$

We first prove that the agent whose preference is $\left\{F_{1}, F_{2}\right\}$ will not cheat. Suppose $p_{i}=\left\{F_{1}, F_{2}\right\}$. We assume that $s_{2}^{\prime}$ is closer to agent $i$ than $s_{1}^{\prime}$. (The other case is the same except the tie breaking which does not affect the proof much.) Therefore, agent $i$ has incentive to cheat only if Mechanism 1 's output is $\left(s_{1}^{\prime}, s_{1}^{\prime}\right)$. Let $\boldsymbol{p}$ be the preference profile where no agent cheats and $\boldsymbol{p}^{\prime}$ be the preference profile where agent $i$ misreports his preference as $\left\{F_{1}\right\}$. Table 1 shows the difference in social cost for $\boldsymbol{p}^{\prime}$ and $\boldsymbol{p}$.

Since Mechanism 1's output is $\left(s_{1}^{\prime}, s_{1}^{\prime}\right)$ for preference profile $\boldsymbol{p}, S C_{\boldsymbol{x}, \boldsymbol{p}}\left(s_{1}^{\prime}, s_{1}^{\prime}\right)$ is the minimum among those four social costs for $\boldsymbol{p}$. By Table 1 , we can see that $S C_{\boldsymbol{x}, \boldsymbol{p}^{\prime}}\left(s_{1}^{\prime}, s_{1}^{\prime}\right)$ is the minimum among those four social costs for $\boldsymbol{p}^{\prime}$. This shows that Mechanism 1's output will still be $\left(s_{1}^{\prime}, s_{1}^{\prime}\right)$ even if agent $i$ misreports his preference as $\left\{F_{1}\right\}$. Therefore, agent $i$ has no incentive to misreport his preference as $\left\{F_{1}\right\}$. Using the same analysis, we can prove that agent $i$ has no incentive to misreport his preference as $\left\{F_{2}\right\}$. Hence, agent $i$ has no incentive to cheat.

We then prove that the agent whose preference is $\left\{F_{1}\right\}$ or $\left\{F_{2}\right\}$ will not cheat. Suppose that $p_{i}=\left\{F_{1}\right\}$. (Using a similar analysis we can prove the case that $p_{i}=\left\{F_{2}\right\}$.) Again, We just assume that $s_{2}^{\prime}$ is closer to agent $i$ than $s_{1}^{\prime}$ since the proof for the other case is similar. Hence, agent $i$ might cheat only when $F_{1}$ is built at $s_{1}^{\prime}$. At this time, $F_{2}$ may be built at $s_{1}^{\prime}$ or $s_{2}^{\prime}$. Let $\boldsymbol{p}$ be the preference profile where no agent cheats, $\boldsymbol{p}^{\prime}$ be the preference profile where agent $i$ misreports his preference as $\left\{F_{2}\right\}$ and $\boldsymbol{p}^{\prime \prime}$ be the preference profile where agent $i$ misreports his preference as $\left\{F_{1}, F_{2}\right\}$. Table 2 shows the difference in social cost for $\boldsymbol{p}^{\prime}$ and $\boldsymbol{p}$ and for $\boldsymbol{p}^{\prime \prime}$ and $\boldsymbol{p}$.

If $F_{2}$ is built at $s_{1}^{\prime}$ for $\boldsymbol{p}$, then

$$
S C_{\boldsymbol{x}, \boldsymbol{p}}\left(s_{1}^{\prime}, s_{1}^{\prime}\right) \leq \min \left\{S C_{\boldsymbol{x}, \boldsymbol{p}}\left(s_{2}^{\prime}, s_{1}^{\prime}\right), S C_{\boldsymbol{x}, \boldsymbol{p}}\left(s_{2}^{\prime}, s_{2}^{\prime}\right)\right\} .
$$

By Table 2, we can see that

$$
S C_{\boldsymbol{x}, \boldsymbol{p}^{\prime}}\left(s_{1}^{\prime}, s_{1}^{\prime}\right) \leq \min \left\{S C_{\boldsymbol{x}, \boldsymbol{p}^{\prime}}\left(s_{2}^{\prime}, s_{1}^{\prime}\right), S C_{\boldsymbol{x}, \boldsymbol{p}^{\prime}}\left(s_{2}^{\prime}, s_{2}^{\prime}\right)\right\},
$$

\begin{tabular}{|c|c|}
\hline$\left(y_{1}, y_{2}\right)$ & $S C_{\boldsymbol{x}, \boldsymbol{p}^{\prime}}\left(y_{1}, y_{2}\right)-S C_{\boldsymbol{x}, \boldsymbol{p}}\left(y_{1}, y_{2}\right)$ \\
\hline$\left(s_{1}^{\prime}, s_{1}^{\prime}\right)$ & 0 \\
\hline$\left(s_{1}^{\prime}, s_{2}^{\prime}\right)$ & $d\left(x_{i}, s_{2}^{\prime}\right)-d\left(x_{i}, s_{1}^{\prime}\right) \leq 0$ \\
\hline$\left(s_{2}^{\prime}, s_{1}^{\prime}\right)$ & $d\left(x_{i}, s_{1}^{\prime}\right)-d\left(x_{i}, s_{2}^{\prime}\right) \geq 0$ \\
\hline$\left(s_{2}^{\prime}, s_{2}^{\prime}\right)$ & 0 \\
\hline \hline$\left(y_{1}, y_{2}\right)$ & $S C_{\boldsymbol{x}, \boldsymbol{p}^{\prime \prime}}\left(y_{1}, y_{2}\right)-S C_{\boldsymbol{x}, \boldsymbol{p}}\left(y_{1}, y_{2}\right)$ \\
\hline$\left(s_{1}^{\prime}, s_{1}^{\prime}\right)$ & 0 \\
\hline$\left(s_{1}^{\prime}, s_{2}^{\prime}\right)$ & $d\left(x_{i}, s_{2}^{\prime}\right)-d\left(x_{i}, s_{1}^{\prime}\right) \leq 0$ \\
\hline$\left(s_{2}^{\prime}, s_{1}^{\prime}\right)$ & 0 \\
\hline$\left(s_{2}^{\prime}, s_{2}^{\prime}\right)$ & 0 \\
\hline
\end{tabular}

Table 2: the difference in social cost for $\boldsymbol{p}^{\prime}$ and $\boldsymbol{p}$ and for $\boldsymbol{p}^{\prime \prime}$ and $\boldsymbol{p}$ 


$$
S C_{\boldsymbol{x}, \boldsymbol{p}^{\prime \prime}}\left(s_{1}^{\prime}, s_{1}^{\prime}\right) \leq \min \left\{S C_{\boldsymbol{x}, \boldsymbol{p}^{\prime \prime}}\left(s_{2}^{\prime}, s_{1}^{\prime}\right), S C_{\boldsymbol{x}, \boldsymbol{p}^{\prime \prime}}\left(s_{2}^{\prime}, s_{2}^{\prime}\right)\right\}
$$

This means $F_{1}$ will not be built at $s_{2}^{\prime}$ even if agent $i$ misreports his preference as $\left\{F_{2}\right\}$ or $\left\{F_{1}, F_{2}\right\}$.

If $F_{2}$ is built at $s_{2}^{\prime}$ for $\boldsymbol{p}$, then

$$
S C_{\boldsymbol{x}, \boldsymbol{p}}\left(s_{1}^{\prime}, s_{2}^{\prime}\right) \leq \min \left\{S C_{\boldsymbol{x}, \boldsymbol{p}}\left(s_{2}^{\prime}, s_{1}^{\prime}\right), S C_{\boldsymbol{x}, \boldsymbol{p}}\left(s_{2}^{\prime}, s_{2}^{\prime}\right)\right\} .
$$

By Table 2, we can see that

$$
\begin{aligned}
& S C_{\boldsymbol{x}, \boldsymbol{p}^{\prime}}\left(s_{1}^{\prime}, s_{2}^{\prime}\right) \leq \min \left\{S C_{\boldsymbol{x}, \boldsymbol{p}^{\prime}}\left(s_{2}^{\prime}, s_{1}^{\prime}\right), S C_{\boldsymbol{x}, \boldsymbol{p}^{\prime}}\left(s_{2}^{\prime}, s_{2}^{\prime}\right)\right\}, \\
& S C_{\boldsymbol{x}, \boldsymbol{p}^{\prime \prime}}\left(s_{1}^{\prime}, s_{2}^{\prime}\right) \leq \min \left\{S C_{\boldsymbol{x}, \boldsymbol{p}^{\prime \prime}}\left(s_{2}^{\prime}, s_{1}^{\prime}\right), S C_{\boldsymbol{x}, \boldsymbol{p}^{\prime \prime}}\left(s_{2}^{\prime}, s_{2}^{\prime}\right)\right\} .
\end{aligned}
$$

Similarly, this means that agent $i$ 's misreporting will not make $F_{1}$ closer. Therefore agent $i$ has no incentive to cheat.

\section{Analysis of the Approximation Ratio}

\subsection{Preparation}

Before we analyze the approximation ratio of Mechanism 1, we introduce some lemmas.

Lemma 1. Denote Mechanism 1 by $M$. Suppose there is an instance $(\boldsymbol{x}, \boldsymbol{p})$ such that $S C_{\boldsymbol{x}, \boldsymbol{p}}(M(\boldsymbol{x}, \boldsymbol{p}))=\alpha$. $\min _{y_{1}, y_{2}} S C_{\boldsymbol{x}, \boldsymbol{p}}\left(y_{1}, y_{2}\right)$ and $p_{i}=\left\{F_{1}, F_{2}\right\}$. Then there exists a preference profile $\tilde{\boldsymbol{p}}$ which differs only at agent $i$ 's preference such that $\tilde{p}_{i} \neq\left\{F_{1}, F_{2}\right\}$ and $S C_{\boldsymbol{x}, \tilde{\boldsymbol{p}}}(M(\boldsymbol{x}, \tilde{\boldsymbol{p}})) \geq$ $\alpha \cdot \min _{y_{1}, y_{2}} S C_{\boldsymbol{x}, \tilde{\boldsymbol{p}}}\left(y_{1}, y_{2}\right)$.

Proof. Suppose $(\boldsymbol{x}, \boldsymbol{p})$ is an instance as above. Let $\left(o p t_{1}, o p t_{2}\right)$ be the locations such that $S C_{\boldsymbol{x}, \boldsymbol{p}}\left(o p t_{1}, o p t_{2}\right)=$ $\min _{y_{1}, y_{2}} S C_{\boldsymbol{x}, \boldsymbol{p}}\left(y_{1}, y_{2}\right)$. Define

$$
\tilde{p}_{i}=\left\{\begin{array}{l}
\left\{F_{1}\right\}, \text { if } d\left(x_{i}, o p t_{1}\right)<d\left(x_{i}, o p t_{2}\right) \\
\left\{F_{2}\right\}, \text { otherwise }
\end{array} .\right.
$$

Let $\tilde{\boldsymbol{p}}$ be the preference profile obtained by replacing $p_{i}$ with $\tilde{p}_{i}$ in $\boldsymbol{p}$.

We first prove $S C_{\boldsymbol{x}, \boldsymbol{p}}(M(\boldsymbol{x}, \boldsymbol{p})) \leq S C_{\boldsymbol{x}, \tilde{\boldsymbol{p}}}(M(\boldsymbol{x}, \tilde{\boldsymbol{p}}))$. Since the location profiles in $(\boldsymbol{x}, \boldsymbol{p})$ and $(\boldsymbol{x}, \tilde{\boldsymbol{p}})$ are the same, $\left(s_{1}^{\prime}, s_{2}^{\prime}\right)$ in $M(\boldsymbol{x}, \boldsymbol{p})$ and $M(\boldsymbol{x}, \tilde{\boldsymbol{p}})$ are the same. Note that for any $\left(y_{1}, y_{2}\right)$ we have $S C_{\boldsymbol{x}, \boldsymbol{p}}\left(y_{1}, y_{2}\right) \leq S C_{\boldsymbol{x}, \tilde{\boldsymbol{p}}}\left(y_{1}, y_{2}\right)$, since $\tilde{p}_{i} \subseteq p_{i}$ and other agents' preferences are the same. Therefore, we have $S C_{\boldsymbol{x}, \boldsymbol{p}}\left(y_{1}, y_{2}\right) \leq S C_{\boldsymbol{x}, \tilde{\boldsymbol{p}}}\left(y_{1}, y_{2}\right)$ for $y_{1}, y_{2} \in$ $\left\{s_{1}^{\prime}, s_{2}^{\prime}\right\}$, which implies

$$
\begin{aligned}
& S C_{\boldsymbol{x}, \boldsymbol{p}}(M(\boldsymbol{x}, \boldsymbol{p}))=\min _{y_{1}, y_{2} \in\left\{s_{1}^{\prime}, s_{2}^{\prime}\right\}} S C_{\boldsymbol{x}, \boldsymbol{p}}\left(y_{1}, y_{2}\right) \\
\leq & \min _{y_{1}, y_{2} \in\left\{s_{1}^{\prime}, s_{2}^{\prime}\right\}} S C_{\boldsymbol{x}, \tilde{\boldsymbol{p}}}\left(y_{1}, y_{2}\right)=S C_{\boldsymbol{x}, \tilde{\boldsymbol{p}}}(M(\boldsymbol{x}, \tilde{\boldsymbol{p}})) .
\end{aligned}
$$

We then prove $\min _{y_{1}, y_{2}} S C_{\boldsymbol{x}, \boldsymbol{p}}\left(y_{1}, y_{2}\right) \geq \min _{y_{1}, y_{2}} S C_{\boldsymbol{x}, \tilde{\boldsymbol{p}}}\left(y_{1}, y_{2}\right)$. By the definition of $\tilde{p}_{i}$, we have

$$
S C_{\boldsymbol{x}, \tilde{\boldsymbol{p}}}\left(o p t_{1}, o p t_{2}\right)=S C_{\boldsymbol{x}, \boldsymbol{p}}\left(o p t_{1}, o p t_{2}\right) .
$$

Therefore, we have

$$
\begin{aligned}
& \min _{y_{1}, y_{2}} S C_{\boldsymbol{x}, \tilde{\boldsymbol{p}}}\left(y_{1}, y_{2}\right) \leq S C_{\boldsymbol{x}, \tilde{\boldsymbol{p}}}\left(o p t_{1}, o p t_{2}\right) \\
= & S C_{\boldsymbol{x}, \boldsymbol{p}}\left(o p t_{1}, o p t_{2}\right)=\min _{y_{1}, y_{2}} S C_{\boldsymbol{x}, \boldsymbol{p}}\left(y_{1}, y_{2}\right) .
\end{aligned}
$$

Thus, we have $S C_{\boldsymbol{x}, \tilde{\boldsymbol{p}}}(M(\boldsymbol{x}, \tilde{\boldsymbol{p}})) \geq S C_{\boldsymbol{x}, \boldsymbol{p}}(M(\boldsymbol{x}, \boldsymbol{p}))=\alpha$. $\min _{y_{1}, y_{2}} S C_{\boldsymbol{x}, \boldsymbol{p}}\left(y_{1}, y_{2}\right) \geq \alpha \cdot \min _{y_{1}, y_{2}} S C_{\boldsymbol{x}, \tilde{\boldsymbol{p}}}\left(y_{1}, y_{2}\right)$.
Since analyzing the approximation ratio is just finding an instance with maximum $\alpha$ as above, by Lemma 1 and simple induction, we can assume $p_{i} \neq\left\{F_{1}, F_{2}\right\}$ for every $i \in\{1,2, \ldots, n\}$ when we analyze the approximation ratio of Mechanism 1.

Lemma 2. $S C_{\boldsymbol{x}, \boldsymbol{q}}\left(s_{1}^{\prime}, s_{2}^{\prime}\right) \leq \alpha \cdot \min _{y_{1}, y_{2}} S C_{\boldsymbol{x}, \boldsymbol{p}}\left(y_{1}, y_{2}\right)$ holds for any location profile $\boldsymbol{x}$ and preference profile $\boldsymbol{p}$.

Proof. Since $S C_{\boldsymbol{x}, \boldsymbol{q}}\left(s_{1}^{\prime}, s_{2}^{\prime}\right)$ gives an $\alpha$-approximation for $S C_{\boldsymbol{x}, \boldsymbol{q}}\left(s_{1}, s_{2}\right)$, we have

$$
\begin{aligned}
& S C_{\boldsymbol{x}, \boldsymbol{q}}\left(s_{1}^{\prime}, s_{2}^{\prime}\right) \leq \alpha \cdot S C_{\boldsymbol{x}, \boldsymbol{q}}\left(s_{1}, s_{2}\right) \\
= & \alpha \cdot \min _{y_{1}, y_{2}} S C_{\boldsymbol{x}, \boldsymbol{q}}\left(y_{1}, y_{2}\right) \leq \alpha \cdot \min _{y_{1}, y_{2}} S C_{\boldsymbol{x}, \boldsymbol{p}}\left(y_{1}, y_{2}\right) .
\end{aligned}
$$

\subsection{Analysis of the Approximation Ratio of $1+2 \alpha$}

We first introduce some notations to simplify the description of later analysis.

Given location profile $\boldsymbol{x}$ and preference profile $\boldsymbol{p}$, by Lemma 1, we can divide all agents into two sets $\left\{i \mid p_{i}=\right.$ $\left.\left\{F_{1}\right\}\right\}$ and $\left\{i \mid p_{i}=\left\{F_{2}\right\}\right\}$. Let $o p t_{1}$ be the geometric median of multiset $S_{1}=\left\{x_{i} \mid p_{i}=\left\{F_{1}\right\}\right\}$ and $o p t_{2}$ be the geometric median of multiset $S_{2}=\left\{x_{i} \mid p_{i}=\left\{F_{2}\right\}\right\}$. Then we have $S C_{\boldsymbol{x}, \boldsymbol{p}}\left(\right.$ opt $\left._{1}, \mathrm{opt}_{2}\right)=\min _{y_{1}, y_{2}} S C_{\boldsymbol{x}, \boldsymbol{p}}\left(y_{1}, y_{2}\right)$.

Given point set $S$ and point $v$, we define $d(S, v)=$ $\sum_{u \in S} d(u, v)$. (This definition differs from the common one where $d(S, v)$ is defined as $\min _{u \in S} d(u, v)$.) We define some notations as follows.

- $S C_{k} \triangleq \min \left\{d\left(S_{k}, s_{1}^{\prime}\right), d\left(S_{k}, s_{2}^{\prime}\right)\right\}, k=1,2$. $S C \triangleq S C_{1}+S C_{2}$

- $O P T_{k} \triangleq d\left(S_{k}, o p t_{k}\right), k=1,2$. $O P T \triangleq O P T_{1}+O P T_{2}$.

- $B E S T_{k} \triangleq \sum_{x \in S_{k}} \min \left\{d\left(x, s_{1}^{\prime}\right), d\left(x, s_{2}^{\prime}\right)\right\}, k=1,2$. $B E S T \triangleq B E S T_{1}+B E S T_{2}$.

Intuitively, $S C$ is equal to the social cost calculated by Mechanism 1, i.e. $S C_{\boldsymbol{x}, \boldsymbol{p}}(M(\boldsymbol{x}, \boldsymbol{p})) . \quad O P T$ is equal to the minimum social cost for $\boldsymbol{p}$, i.e. $\min _{y_{1}, y_{2}} S C_{\boldsymbol{x}, \boldsymbol{p}}\left(y_{1}, y_{2}\right)$. $B E S T$ is equal to the approximated minimum social cost for $\boldsymbol{q}$, i.e. $S C_{\boldsymbol{x}, \boldsymbol{q}}\left(s_{1}^{\prime}, s_{2}^{\prime}\right)$. ( $\boldsymbol{q}$ is defined in Mechanism 1.) With these notations, Lemma 2 implies $B E S T \leq \alpha \cdot O P T$.

Now, we introduce our result on the approximation ratio of Mechanism 1.

Theorem 2. Mechanism 1 has an approximation ratio of $1+$ $2 \alpha$, i.e. $S C \leq(1+2 \alpha) \cdot O P T$.

Proof. We try to prove $S C-O P T \leq 2 \cdot B E S T$. It suffices to show $S C_{k}-O P T_{k} \leq 2 \cdot B E S T_{k}, k=1,2$. By symmetry, we only show $S C_{1}-O P T_{1} \leq 2 \cdot B E S T_{1}$.

We divide $S_{1}$ into $A$ and $\bar{B}$ using the following rule. For each $x \in S_{1}$, if $d\left(x, s_{1}^{\prime}\right)<d\left(x, s_{2}^{\prime}\right)$, then let $x \in A$. Otherwise, let $x \in B$. Without loss of generality, suppose that $|A| \geq|B|$. Observe that for any two agents' locations $x$ and $y$, by triangle inequality we have

$$
\begin{aligned}
& d\left(x, s_{1}^{\prime}\right)+d\left(y, s_{1}^{\prime}\right)-\left(d\left(x, o p t_{1}\right)+d\left(y, o p t_{1}\right)\right) \\
\leq & d\left(x, s_{1}^{\prime}\right)+d\left(y, s_{1}^{\prime}\right)-d(x, y) \leq 2 \cdot d\left(x, s_{1}^{\prime}\right) .
\end{aligned}
$$


Hence, we divide $A$ into $A_{1}$ and $A_{2}$ such that $\left|A_{1}\right|=|B|$ and $A=A_{1} \cup A_{2}$ and match each location in $B$ with a location in $A_{1}$. Using the observation above, we have

$$
\begin{aligned}
& S C_{1}-O P T_{1} \leq d\left(S_{1}, s_{1}^{\prime}\right)-d\left(S_{1}, o p t_{1}\right) \\
\leq & 2 \cdot d\left(A_{1}, s_{1}^{\prime}\right)+\left(d\left(A_{2}, s_{1}^{\prime}\right)-d\left(A_{2}, o p t_{1}\right)\right) \\
\leq & 2 \cdot d\left(A, s_{1}^{\prime}\right) \leq 2 \cdot B E S T_{1} .
\end{aligned}
$$

By Lemma 2, we have $S C-O P T \leq 2 \cdot B E S T \leq 2 \alpha$. $O P T$. Hence, $S C \leq(1+2 \alpha) \cdot O P T$.

Consider the following two special metric spaces:

- The metric space is $\mathbb{R}^{2}$. In this case, for any fixed $\epsilon>0$, by [Arora et al., 1998] we have a $(1+\epsilon / 2)$ approximation polynomial time algorithm for 2-median problem, i.e. $\alpha=1+\epsilon / 2$. Thus, Mechanism 1 guarantees an approximation of $3+\epsilon$.

- The metric space is a line, i.e. $\mathbb{R}$. In this case, it is easy to find exact 2-median in polynomial time. Thus, Mechanism 1 guarantees an approximation of 3, which is a substantial improvement given the best result achieved by [Yuan et al., 2016] is $n / 2+1$.

\subsection{Improvement for Line Metric}

In this section, we only consider the line metric case, i.e. the metric space is $\mathbb{R}$. For simplicity of later analysis, let $\Delta_{k}=$ $\left(S C_{k}-O P T_{k}\right) / 2, k=1,2$ and $\Delta=\Delta_{1}+\Delta_{2}$. In this case, we can calculate exact $s_{1}$ and $s_{2}$ in polynomial time, thus we just use $s_{\ell}$ and $s_{r}$ in the analysis instead and suppose $s_{\ell} \leq s_{\text {mid }} \leq s_{r}$ where $s_{\text {mid }}$ is the midpoint of $s_{\ell}$ and $s_{r}$. Since the metric is $\mathbb{R}$, opt $t_{1}$ and $o p t_{2}$ are the median values of $S_{1}$ and $S_{2}$. Without loss of generality, we can assume both $\left|S_{1}\right|$ and $\left|S_{2}\right|$ are even. (Otherwise we just double the number of agents by making a copy of original agents. This does not change the approximation ratio.)

We observe that our analysis can be tightened by doing some trade-off so as to get the following result.

Theorem 3. Mechanism 1 has an approximation ratio of 2.75, i.e. $S C \leq 2.75 \cdot O P T$.

Proof. We bound $\Delta_{1}$ first. By symmetry, we assume that $o p t_{1} \leq s_{\text {mid }}$. Let $s_{c}$ be the point satisfying $d\left(s_{c}, s_{m i d}\right)=$ $c \cdot d\left(s_{\ell}, s_{c}\right)$. Here, $c$ is a constant parameter to be determined later.

We analyze in the following three cases, according to the position of $o p t_{1}$.

Case 1. $o p t_{1} \leq s_{\ell}$.

In this case, we divide $S_{1}$ into four parts: $X_{1}, X_{2}, X_{3}$ and $X_{4}$.

1. $X_{1}$ contains the points on the left of $o p t_{1}$.

2. $X_{2}$ contains the points between $o p t_{1}$ and $s_{\ell}$.

3. $X_{3}$ contains the points between $s_{\ell}$ and $s_{m i d}$.

4. $X_{4}$ contains the points on the right of $s_{m i d}$.

We allocate points on the boundary in such a way that $\left|X_{1}\right|=\left|X_{2}\right|+\left|X_{3}\right|+\left|X_{4}\right|$ (This can be achieved since $o p t_{1}$ divides $S_{1}$ into two parts, left part and right part, of the same size).
Since $o p t_{1} \leq s_{\ell}$, we find that $\Delta_{1}$ is equal to the distance between $s_{\ell}$ and points in $S_{1}$ which lie between $o p t_{1}$ and $s_{\ell}$, i.e. $d\left(X_{2}, s_{\ell}\right)$. Hence, we have

$$
\begin{aligned}
\Delta_{1} & =d\left(X_{2}, s_{\ell}\right) \leq\left|X_{2}\right| \cdot d\left(o_{1} t_{1}, s_{\ell}\right) \\
& \leq\left|X_{1}\right| \cdot d\left(o_{1}, s_{\ell}\right) \leq d\left(X_{1}, s_{\ell}\right) .
\end{aligned}
$$

In this case, we have

$$
\begin{aligned}
\operatorname{BEST}_{1} & =d\left(X_{1} \cup X_{2} \cup X_{3}, s_{\ell}\right)+d\left(X_{4}, s_{r}\right) \\
& \geq d\left(X_{1}, s_{\ell}\right)+d\left(X_{2}, s_{\ell}\right) \geq 2 \cdot \Delta_{1} .
\end{aligned}
$$

This implies $\Delta_{1} \leq 1 / 2 \cdot B E S T_{1}$.

Case 2. $s_{c} \leq o p t_{1} \leq s_{m i d}$

In this case, we divide $S_{1}$ into five parts: $X_{1}, X_{2}, X_{3}, X_{4}$ and $X_{5}$.

1. $X_{1}$ contains the points between $s_{\ell}$ and $o p t_{1}$.

2. $X_{2}$ contains the points between $s_{m i d}$ and $s_{r}$.

3. $X_{3}$ contains the points between $o p t_{1}$ and $s_{m i d}$.

4. $X_{4}$ contains the points on the left of $s_{\ell}$.

5. $X_{5}$ contains the points on the right of $s_{r}$.

Similarly, we can assume $\left|X_{1}\right|+\left|X_{4}\right|=\left|X_{2}\right|+\left|X_{3}\right|+\left|X_{5}\right|$.

By the definition of $\Delta_{1}$, using a similar proof as Theorem 2 , we have

$$
\Delta_{1}=\min \left\{d\left(X_{1}, s_{\ell}\right), d\left(X_{3}, s_{r}\right)+d\left(X_{2}, s_{r}\right)\right\} .
$$

In this case, we have

$$
\begin{aligned}
B E S T_{1} & =d\left(X_{1} \cup X_{3} \cup X_{4}, s_{\ell}\right)+d\left(X_{2} \cup X_{5}, s_{r}\right) \\
& \geq d\left(X_{1}, s_{\ell}\right)+d\left(X_{2}, s_{r}\right)+d\left(X_{3}, s_{\ell}\right) .
\end{aligned}
$$

Since $d\left(s_{c}, s_{\text {mid }}\right)=c \cdot d\left(s_{\ell}, s_{c}\right)$ and $d\left(s_{\ell}, s_{\text {mid }}\right)=$ $d\left(s_{\text {mid }}, s_{r}\right)$, we have $d\left(s_{c}, s_{r}\right)=(2 c+1) \cdot d\left(s_{\ell}, s_{c}\right)$. Given that ${ }^{o p t} t_{1} \geq s_{c}$ in this case, $d\left(v, s_{r}\right) \leq(2 c+1) \cdot d\left(v, s_{\ell}\right)$ holds for every $v \in X_{3}$. Thus, we have $d\left(X_{3}, s_{r}\right) \leq$ $(2 c+1) \cdot d\left(X_{3}, s_{\ell}\right)$. Hence,

$$
\begin{aligned}
& d\left(X_{3}, s_{r}\right)+d\left(X_{2}, s_{r}\right) \\
\leq & (2 c+1) \cdot d\left(X_{3}, s_{\ell}\right)+d\left(X_{2}, s_{r}\right) \\
\leq & (2 c+1) \cdot\left(d\left(X_{3}, s_{\ell}\right)+d\left(X_{2}, s_{r}\right)\right) .
\end{aligned}
$$

If $d\left(X_{1}, s_{\ell}\right) \leq(2 c+1) /(2 c+2) \cdot B E S T_{1}$, then

$$
\Delta_{1} \leq d\left(X_{1}, s_{\ell}\right) \leq \frac{2 c+1}{2 c+2} B E S T_{1}
$$

Otherwise, $d\left(X_{1}, s_{\ell}\right)>(2 c+1) /(2 c+2) \cdot B E S T_{1}$. Then,

$$
\begin{aligned}
\Delta_{1} & \leq d\left(X_{3}, s_{r}\right)+d\left(X_{2}, s_{r}\right) \\
& \leq(2 c+1) \cdot\left(d\left(X_{3}, s_{\ell}\right)+d\left(X_{2}, s_{r}\right)\right) \\
& \leq(2 c+1) \cdot\left(B E S T_{1}-d\left(X_{1}, s_{\ell}\right)\right) \\
& <\frac{2 c+1}{2 c+2} B E S T_{1} .
\end{aligned}
$$

Therefore, in this case, we have $\Delta_{1} \leq(2 c+1) /(2 c+2)$. $B E S T_{1}$.

Case 3. $s_{\ell}<o p t_{1}<s_{c}$. 
In this case, we divide $S_{1}$ into the same five parts defined in Case 2. Similar to Case 2, we have

$$
\begin{aligned}
& \Delta_{1}=\min \left\{d\left(X_{1}, s_{\ell}\right), d\left(X_{3}, s_{r}\right)+d\left(X_{2}, s_{r}\right)\right\}, \\
& B E S T_{1}=d\left(X_{1} \cup X_{3} \cup X_{4}, s_{\ell}\right)+d\left(X_{2} \cup X_{5}, s_{r}\right) .
\end{aligned}
$$

Let $\beta$ be a constant parameter in $(0,1)$. We will determine the value of $\beta$ later.

If $d\left(X_{2}, s_{r}\right)+d\left(X_{3}, s_{\ell}\right) \geq \beta \cdot d\left(X_{1}, s_{\ell}\right)$, we have

$$
\begin{aligned}
B E S T_{1} & \geq d\left(X_{1}, s_{\ell}\right)+d\left(X_{2}, s_{r}\right)+d\left(X_{3}, s_{\ell}\right) \\
& \geq(1+\beta) \cdot d\left(X_{1}, s_{\ell}\right) \\
& \geq(1+\beta) \cdot \Delta_{1} .
\end{aligned}
$$

This implies that $\Delta_{1} \leq 1 /(1+\beta) \cdot B E S T_{1}$.

Otherwise, $d\left(X_{2}, s_{r}\right)+d\left(X_{3}, s_{\ell}\right)<\beta \cdot d\left(X_{1}, s_{\ell}\right)$. Then we have

$$
\begin{aligned}
\beta \cdot d\left(X_{1}, s_{\ell}\right) & >d\left(X_{2}, s_{r}\right)+d\left(X_{3}, s_{\ell}\right) \\
& \geq d\left(X_{2}, s_{r}\right)+\left|X_{3}\right| \cdot d\left(\text { opt }_{1}, s_{\ell}\right) \\
& \geq d\left(X_{2}, s_{r}\right)+\frac{\left|X_{3}\right|}{\left|X_{1}\right|} \cdot d\left(X_{1}, s_{\ell}\right) .
\end{aligned}
$$

Now we use $O P T_{1}$ to bound $\Delta_{1}$. By definition, we have

$$
O P T_{1}=d\left(S_{1}, o p t_{1}\right) \geq d\left(X_{2}, o p t_{1}\right)+d\left(X_{5}, o p t_{1}\right) .
$$

Note that $d\left(v, o p t_{1}\right)=d\left(o p t_{1}, s_{r}\right)-d\left(v, s_{r}\right)$ holds for every $v \in X_{2}$ and $d\left(v, o p t_{1}\right) \geq d\left(o p t_{1}, s_{r}\right)$ holds for every $v \in X_{5}$. Hence, we have

$$
\begin{aligned}
& O P T_{1} \\
\geq & \left|X_{2}\right| \cdot d\left(o p t_{1}, s_{r}\right)-d\left(X_{2}, s_{r}\right)+\left|X_{5}\right| \cdot d\left(o p t_{1}, s_{r}\right) \\
= & \left(\left|X_{2}\right|+\left|X_{5}\right|\right) \cdot d\left(o p t_{1}, s_{r}\right)-d\left(X_{2}, s_{r}\right) \\
\geq & \left(\left|X_{2}\right|+\left|X_{5}\right|\right) \cdot \frac{2 c+1}{2 c+2} \cdot d\left(s_{\ell}, s_{r}\right)-d\left(X_{2}, s_{r}\right) .
\end{aligned}
$$

Note that we have $d\left(X_{1}, s_{\ell}\right) \leq\left|X_{1}\right| \cdot d\left(s_{\ell}\right.$, opt $\left._{1}\right) \leq\left|X_{1}\right|$. $d\left(s_{\ell}, s_{r}\right) /(2 c+2)$. Hence,

$$
\begin{aligned}
& O P T_{1} \\
\geq & (2 c+1) \cdot \frac{\left|X_{2}\right|+\left|X_{5}\right|}{\left|X_{1}\right|} \cdot d\left(X_{1}, s_{\ell}\right)-d\left(X_{2}, s_{r}\right) \\
\geq & (2 c+1) \cdot \frac{\left|X_{1}\right|-\left|X_{3}\right|}{\left|X_{1}\right|} \cdot d\left(X_{1}, s_{\ell}\right)-d\left(X_{2}, s_{r}\right) \\
= & (2 c+1) \cdot d\left(X_{1}, s_{\ell}\right) \\
& -\left((2 c+1) \cdot \frac{\left|X_{3}\right|}{\left|X_{1}\right|} \cdot d\left(X_{1}, s_{\ell}\right)+d\left(X_{2}, s_{r}\right)\right) \\
\geq & (2 c+1) \cdot d\left(X_{1}, s_{\ell}\right) \\
& -(2 c+1)\left(\frac{\left|X_{3}\right|}{\left|X_{1}\right|} \cdot d\left(X_{1}, s_{\ell}\right)+d\left(X_{2}, s_{r}\right)\right) \\
\geq & (2 c+1) \cdot d\left(X_{1}, s_{\ell}\right)-(2 c+1) \cdot \beta \cdot d\left(X_{1}, s_{\ell}\right) \\
= & (2 c+1) \cdot(1-\beta) \cdot d\left(X_{1}, s_{\ell}\right) \\
\geq & (2 c+1) \cdot(1-\beta) \cdot \Delta_{1} .
\end{aligned}
$$

This means that we have

$$
\Delta_{1} \leq \frac{1}{(2 c+1) \cdot(1-\beta)} O P T_{1} .
$$

We summarize those three cases. Let $c=1$ and $\beta=$ $1 /(2 c+1)=1 / 3$. At least one of the following will happen:

$$
\Delta_{1} \leq \max \left\{\frac{1}{2}, \frac{2 c+1}{2 c+2}, \frac{1}{1+\beta}\right\} \cdot B E S T_{1}=\frac{3}{4} B E S T_{1},
$$

or

$$
\Delta_{1} \leq \frac{1}{(2 c+1) \cdot(1-\beta)} O P T_{1}=\frac{1}{2} O P T_{1} .
$$

Similarly, we can get the for $\Delta_{2}$ :

$$
\Delta_{2} \leq \frac{3}{4} B E S T_{2} \text { or } \Delta_{2} \leq \frac{1}{2} O P T_{2} .
$$

Since there is no relationship between $O P T_{1}$ and $B E S T_{1}$, we consider $\Delta_{1}$ and $\Delta_{2}$ together, using $\Delta=\Delta_{1}+\Delta_{2}$. Without loss of generality, we assume $B E S T_{1} \geq B E S T_{2}$. We analyze in the following 4 cases:

Case 1: $\Delta_{1} \leq 3 / 4 \cdot B E S T_{1}, \Delta_{2} \leq 3 / 4 \cdot B E S T_{2}$. In this case, we have

$$
\Delta \leq \frac{3}{4}\left(B E S T_{1}+B E S T_{2}\right)=\frac{3}{4} B E S T \leq \frac{3}{4} O P T .
$$

Case 2: $\Delta_{1} \leq 1 / 2 \cdot O P T_{1}, \Delta_{2} \leq 1 / 2 \cdot O P T_{2}$. In this case, we have

$$
\Delta \leq \frac{1}{2}\left(O P T_{1}+O P T_{2}\right)=\frac{1}{2} O P T .
$$

Case 3: $\Delta_{1} \leq 1 / 2 \cdot O P T_{1}, \Delta_{2} \leq 3 / 4 \cdot B E S T_{2}$. In this case, we have

$$
\begin{aligned}
\Delta & \leq \frac{1}{2} O P T_{1}+\frac{3}{4} B_{E S T_{2}} \leq \frac{1}{2} O P T+\frac{3}{8} B E S T \\
& \leq \frac{7}{8} O P T .
\end{aligned}
$$

Case 4: $\Delta_{1} \leq 3 / 4 \cdot B E S T_{1}, \Delta_{2} \leq 1 / 2 \cdot O P T_{2}$. In this case, we have

$$
\begin{aligned}
\Delta & \leq \frac{3}{4} B E S T_{1}+B E S T_{2} \\
& \leq \frac{3}{4} B E S T_{1}+\frac{1}{8} B E S T_{1}+\frac{7}{8} B E S T_{2} \\
& =\frac{7}{8} B E S T \leq \frac{7}{8} O P T .
\end{aligned}
$$

It is easy to see that $\Delta \leq 7 / 8 \cdot O P T$ holds for all these four cases. Therefore, we have $S C-O P T=2 \cdot \Delta \leq 2$. $7 / 8 \cdot O P T=7 / 4 \cdot O P T$, i.e. $S C \leq 2.75 \cdot O P T$.

\subsection{A Lower Bound of the Approximation Ratio of Mechanism 1}

We provide a lower bound for the approximation ratio of Mechanism 1.

Consider the line metric $\mathbb{R}$. Let $N$ be a positive integer. Let $N$ agents be located at $x=0$ with preference $\left\{F_{1}\right\}$, $\lfloor(1+\sqrt{2}) N\rfloor$ agents be located at $x=1$ with preference $\left\{F_{1}\right\}$ and almost infinite (far greater than $N$ ) agents be located at $x=\sqrt{2}$ with preference $\left\{F_{2}\right\}$. We can see that $\left(s_{\ell}, s_{r}\right)$ will be $(0, \sqrt{2})$. Note that the minimum social cost 
is $N$ while the social cost calculated by Mechanism 1 is $\min (\lfloor(1+\sqrt{2}) N\rfloor, \sqrt{2} N+\lfloor(1+\sqrt{2}) N\rfloor(\sqrt{2}-1))$. When $N$ increases to infinity, the ratio of social cost by Mechanism 1 to the minimum social cost approaches $1+\sqrt{2}$. Therefore, $1+\sqrt{2}$ is a lower bound of the approximation ratio of Mechanism 1 .

\section{Generalization}

We have tried to generalize our mechanism to $k$ facilities $(k \geq 3)$. Our generalized mechanism first ignores agents' preferences and assumes all facilities are acceptable for each agent. Then it only uses the location profile to calculate the optimal locations of all $k$ facilities, which we denote as $s_{1}, s_{2}, \ldots, s_{k}$. Finally, the mechanism enumerates $F_{i}$ 's location being at $s_{j}, k^{k}$ cases in total, and chooses the one that minimizes the social cost.

However, unfortunately, this generalized mechanism is not strategyproof when there are more than two facilities. We use an example to show that this mechanism is not able to prevent agents from misreporting their preferences when there are three facilities.

Example 1. Consider the line metric. Suppose that $l_{1}$ and $l_{2}$ are two positive numbers satisfying $2 l_{2}<l_{1}<3 l_{2}$. Let

- two agents whose preferences are $\left\{F_{2}\right\}$ be located at $x=0$,

- one agent whose preference is $\left\{F_{2}\right\}$ be located at $x=$ $l_{1}-l_{2}$,

- one agent whose preference is $\left\{F_{2}\right\}$ be located at $x=l_{1}$,

- one agent whose preference is $\left\{F_{2}, F_{3}\right\}$ be located at $x=l_{1}+l_{2}$,

- almost infinite agents whose preferences are $\left\{F_{1}\right\}$ be located at $x=0$,

- almost infinite agents whose preferences are $\left\{F_{3}\right\}$ be located at $x=2 l_{1}+l_{2}$.

We can see that if we use the generalized mechanism on this instance, $\left(s_{1}, s_{2}, s_{3}\right)$ will be $\left(0, l_{1}, 2 l_{1}+l_{2}\right)$. (See Figure 1.) The almost infinite agents at $s_{1}$ with preferences $\left\{F_{1}\right\}$ force $F_{1}$ to be built at $s_{1}$. (Otherwise, the social cost will be almost infinite.) For the same reason, $F_{3}$ will be built at $s_{3}$.

By enumerating all three possible locations of $F_{2}$ and calculating the social costs, we find that if all agents report their true preferences, then $F_{2}$ will be built at $s_{1}$. However, if the agent at $l_{1}+l_{2}$ (with preference $\left\{F_{2}, F_{3}\right\}$ ) misreports his preference as $\left\{F_{2}\right\}$, then $F_{2}$ will be built at $s_{2}$ and the cost of this

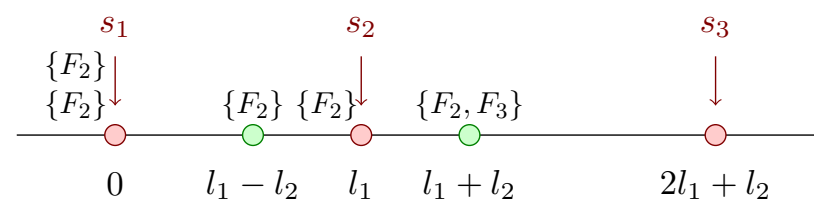

Figure 1: An example which shows the generalized mechanism is not strategyproof. First 5 agents' locations and preferences are illustrated in the figure. agent will decrease from $l_{1}$ to $l_{2}$. Therefore, this generalized mechanism fails to prevent this agent from misreporting his preference.

In this example, we find that the preferred facilities of the agent at $l_{1}+l_{2}$ are built at the second nearest and the third nearest possible location in $\left\{s_{1}, s_{2}, s_{3}\right\}$. By hiding his preference to the facility which is built at the second nearest possible location, the agent increases the social cost and thus the generalized mechanism chooses another solution where another preferred facility of the agent is built at the nearest possible location to the agent. However, this situation will not happen when there are only two facilities because the mechanism chooses the facilities' locations only between two possible locations $s_{1}$ and $s_{2}$. That is the reason why the mechanism is strategyproof when there are only two facilities but not strategyproof for three facilities.

Consider the facility location game with $k$ facilities, where $k>3$. For every $i \in\{4,5, \ldots, k\}$, we add almost infinite agents whose locations are $x=(i-1) \cdot l_{1}+l_{2}$ and preferences are $\left\{F_{i}\right\}$ to the example above. We can see that the new example shows that the generalized mechanism is not strategyproof when there are $k>3$ facilities. Hence, this generalized mechanism is not strategyproof for the facility location game with more than two facilities.

\section{Conclusion}

To summarize, we have designed a strategyproof mechanism whose approximation ratio is no more than $1+2 \alpha$ and no less than $1+\sqrt{2}$, where $\alpha$ is the approximation ratio of the offline optimization version. It is an open question whether the exact approximation ratio of this mechanism for line metric is $1+\sqrt{2}$. Furthermore, the bounds for approximation ratios of strategyproof mechanisms for two facilities do not match yet.

Besides, it is also an interesting open question to design a strategyproof mechanism with constant approximation ratio for the facility location game with three or more facilities.

\section{Acknowledgements}

Yuhao Yao and Jialin Zhang are supported in part by the 973 Program of China Grant No. 2016YFB1000201, the National Natural Science Foundation of China Grants No. 61832003, 61872334, 61761136014.

Minming Li is also from City University of Hong Kong Shenzhen Research Institute, Shenzhen, P. R. China. He is supported by a grant from Research Grants Council of the Hong Kong Special Administrative Region, China (Project No. CityU 11200518) and is partially sponsored by the National Natural Science Foundation of China Grants No.11771365.

Pinyan Lu is supported by Science and Technology Innovation 2030 -"New Generation of Artificial Intelligence" Major Project No. 2018AAA0100903, the National Natural Science Foundation of China Grants No. 61922052, Innovation Program of Shanghai Municipal Education Commission, Program for Innovative Research Team of Shanghai University of Finance and Economics (IRTSHUFE) and the Fundamental Research Funds for the Central Universities. 


\section{References}

[Alon et al., 2010] Noga Alon, Michal Feldman, Ariel D. Procaccia, and Moshe Tennenholtz. Strategyproof approximation of the minimax on networks. Mathematics of Operations Research, 35(3):513-526, 2010.

[Arora et al., 1998] Sanjeev Arora, Prabhakar Raghavan, and Satish Rao. Approximation schemes for euclidean kmedians and related problems. In STOC, volume 98, pages 106-113, 1998.

[Arrow, 1950] Kenneth J Arrow. A difficulty in the concept of social welfare. Journal of political economy, 58(4):328-346, 1950.

[Cai et al., 2016] Qingpeng Cai, Aris Filos-Ratsikas, and Pingzhong Tang. Facility location with minimax envy. In IJCAI, pages 137-143, 2016.

[Chen et al., 2018] Xujin Chen, Xiaodong Hu, Xiaohua Jia, Minming Li, Zhongzheng Tang, and Chenhao Wang. Mechanism design for two-opposite-facility location games with penalties on distance. In $S A G T$, pages 256-260, 2018.

[Cheng et al., 2011] Yukun Cheng, Wei Yu, and Guochuan Zhang. Mechanisms for obnoxious facility game on a path. In COCOA, pages 262-271, 2011.

[Cheng et al., 2013] Yukun Cheng, Wei Yu, and Guochuan Zhang. Strategy-proof approximation mechanisms for an obnoxious facility game on networks. Theoretical Computer Science, 497:154-163, 2013.

[Duan et al., 2019] Lingjie Duan, Bo Li, Minming Li, and Xinping $\mathrm{Xu}$. Heterogeneous two-facility location games with minimum distance requirement. In $A A M A S$, pages 1461-1469, 2019.

[Feigenbaum and Sethuraman, 2015] Itai Feigenbaum and Jay Sethuraman. Strategyproof mechanisms for onedimensional hybrid and obnoxious facility location models. In Workshops at AAAI, 2015.

[Feldman and Wilf, 2013] Michal Feldman and Yoav Wilf. Strategyproof facility lacation and the least squares objective. In $E C$, pages 873-890, 2013.

[Filos-Ratsikas et al., 2017] Aris Filos-Ratsikas, Minming Li, Jie Zhang, and Qiang Zhang. Facility location with double-peaked preferences. Autonomous Agents and Multi-Agent Systems, 31(6):1209-1235, 2017.

[Fong et al., 2018] Ken C.K. Fong, Minming Li, Pinyan Lu, Taiki Todo, and Makoto Yokoo. Facility location games with fractional preferences. In $A A A I$, pages 1039-1046, 2018.

[Fotakis and Tzamos, 2014] Dimitris Fotakis and Christos Tzamos. On the power of deterministic mechanisms for facility location games. ACM Transactions on Economics and Computation, 2(4):15:1-15:37, 2014.

[Fotakis and Tzamos, 2016] Dimitris Fotakis and Christos Tzamos. Strategyproof facility location for concave cost functions. Algorithmica, 76(1):143-167, 2016.
[Gibbard, 1973] Allan Gibbard. Manipulation of voting schemes: a general result. Econometrica: Journal of the Econometric Society, pages 587-601, 1973.

[Ibara and Nagamochi, 2012] Ken Ibara and Hiroshi Nagamochi. Characterizing mechanisms in obnoxious facility game. In COCOA, pages 301-311, 2012.

[Lu et al., 2010] Pinyan Lu, Xiaorui Sun, Yajun Wang, and Zeyuan Allen Zhu. Asymptotically optimal strategy-proof mechanisms for two-facility games. In EC, pages 315$324,2010$.

[Mei et al., 2016] Lili Mei, Minming Li, Deshi Ye, and Guochuan Zhang. Strategy-proof mechanism design for facility location games: Revisited (extended abstract). In $A A M A S$, pages 1463-1464, 2016.

[Mei et al., 2018a] Lili Mei, Deshi Ye, and Guochuan Zhang. Mechanism design for one-facility location game with obnoxious effects on a line. Theoretical Computer Science, 734:46-57, 2018.

[Mei et al., 2018b] Lili Mei, Deshi Ye, and Yong Zhang. Approximation strategy-proof mechanisms for obnoxious facility location on a line. Journal of Combinatorial Optimization, 36(2):549-571, 2018.

[Moulin, 1980] Hervé Moulin. On strategy-proofness and single peakedness. Public Choice, 35(4):437-455, 1980.

[Oomine and Nagamochi, 2016] Morito Oomine and Hiroshi Nagamochi. Characterizing output locations of gsp mechanisms to obnoxious facility game in trees. IEICE Transactions on Information and Systems, 99(3):615-623, 2016.

[Procaccia and Tennenholtz, 2009] Ariel D. Procaccia and Moshe Tennenholtz. Approximate mechanism design without money. In $E C$, pages 177-186, 2009.

[Satterthwaite, 1975] Mark Allen Satterthwaite. Strategyproofness and arrow's conditions: Existence and correspondence theorems for voting procedures and social welfare functions. Journal of economic theory, 10(2):187217, 1975.

[Schummer and Vohra, 2002] James Schummer and Rakesh V. Vohra. Strategy-proof location on a network. Journal of Economic Theory, 104(2):405-428, 2002.

[Serafino and Ventre, 2014] Paolo Serafino and Carmine Ventre. Heterogeneous facility location without money on the line. In ECAI, pages 807-812, 2014.

[Serafino and Ventre, 2015] Paolo Serafino and Carmine Ventre. Truthful mechanisms without money for nonutilitarian heterogeneous facility location. In $A A A I$, pages 1029-1035, 2015.

[Yuan et al., 2016] Hongning Yuan, Kai Wang, Ken CK Fong, Yong Zhang, and Minming Li. Facility location games with optional preference. In ECAI, pages 15201527. IOS Press, 2016.

[Zou and Li, 2015] Shaokun Zou and Minming Li. Facility location games with dual preference. In $A A M A S$, pages 615-623, 2015. 